\title{
EFFECT OF SPOKES STRUCTURES ON CHARACTERISTICS PERFORMANCE OF NON-PNEUMATIC TIRES
}

\author{
A.M. Aboul-Yazid ${ }^{1}$, M.A.A. Emam ${ }^{1}$, S. Shaaban ${ }^{1}$ and M.A. El-Nashar ${ }^{1}$ \\ ${ }^{1}$ Automotive and Tractor Engineering Department \\ Helwan University, Egypt. \\ *Email: genius_cts@hotmail.com \\ Phone: +01067777208; Fax: +202 26332398
}

\begin{abstract}
In this paper, three different configurations of the Michelin's Tweel ${ }^{\mathrm{TM}}$, Resilient Technologies, and Bridgestone non-pneumatic tires (NPTs) are investigated by seeking compliant spoke structures. The quasi-static, two-dimensional analysis focuses on how the contact pressure, vertical tire stiffness and stresses are affected by the spoke structures and shear layer. Two cases of NPTs are investigated: (i) a tire with a composite ring and (ii) a tire without a composite ring. The results of the models were validated by comparison with four computational finite element models. The results showed that the shape of the spokes has a great effect on the tire's behavior in the second case, while the shear layer in the first case reduces the impact of the change of spokes shape especially in contact pressure distribution.
\end{abstract}

Keywords: Non-pneumatic tire (NPT); rolling resistance; contact pressure; FEM.

\section{INTRODUCTION}

The NPT consists of three basic parts including a circular deformable beam, thin elastic collapsible spokes and a rigid hub, as shown in Figure 1. In some designs the polyurethane circular deformable beam may be replaced by rubber, as in the Resilient Technologies NPT [1]. This beam deforms almost entirely due to shear and hence it is called the shear beam and it has a low modulus material [2]. The shear beam consists of a shear layer sandwiched between an inner inextensible membrane (IIEM) and an outer inextensible membrane (OIEM). This beam replaces the inflation pressure and causes low contact pressure. The inextensible membranes are also called reinforcements due to their high circumference stiffness and strength and low radial bending stiffness. This forces the tread rubber to be deformed by shear. Without the reinforcements, the edges of the spokes over the contact zone with the ground would buckle and cause an undesirable non-linear effect of the spokes [3]. NPT design is driven by the critical characteristics of the pneumatic tire, namely mass, stiffness, contact pressure, and rolling resistance. Rolling resistance is one of the main characteristics of interest as it contributes to the fuel consumption of vehicles. Stiffness and contact pressure distribution are other important properties to be addressed when designing an NPT [4]. In the design process, the aim of changing the spoke structure is to determine the values of design variables in order to optimize the performance of the NPT with given constraints. In this study four different spoke structures are used and the effects of different spoke shapes on these four parameters are studied. 


\section{METHODS AND MATERIALS}

\section{Dimensions and Constituent Materials}

The inner ring is made of an aluminum alloy and functions as a rigid hub. The IIEM and OIEM are made of a high strength steel with 1 and $0.75 \mathrm{~mm}$ thickness respectively. Without reinforcements, the edges of the spokes over the contact zone with the ground would buckle and cause an undesirable non-linear effect of the honeycombs [3]. Polyurethane (PU) is used as the constituent material of the spokes and shear layer with $10.2 \mathrm{~mm}$ thickness. PU composite has both elasticity and stiffness at the same time [5]. PU materials have a relatively low modulus that allows for large strain with low stress [6]. The tread component is made of a rubber. The thickness of the tread is set to be 2 $\mathrm{mm}$. The material properties are listed in Table 1. The spokes differ in the four models, as shown in Figure 2. In Michelin's Tweel ${ }^{\mathrm{TM}}$ the spokes have a curvature, thickness and also deviate from the radial line. The spokes are paired and placed at equal intervals along the circumference of the Tweel.

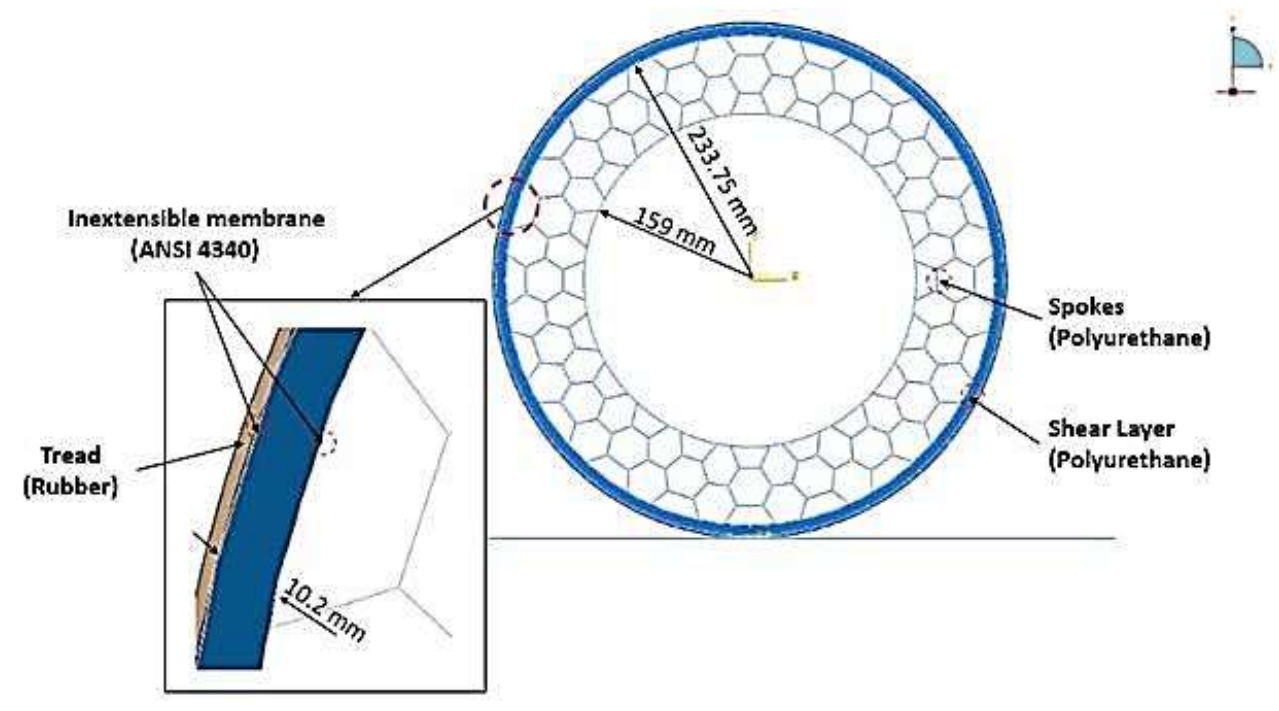

Figure 1. Different parts and dimensions of NPT.

Table 1. Material properties.

\begin{tabular}{ccccc}
\hline Material & $\begin{array}{c}\text { Density, } \\
\rho\left(\mathrm{kg} / \mathrm{m}^{3}\right)\end{array}$ & $\begin{array}{c}\text { Young Modulus, } \\
\mathrm{E}(\mathrm{MPa})\end{array}$ & $\begin{array}{c}\text { Poisson's ratio, } \\
v\end{array}$ & $\begin{array}{c}\text { Shear Modulus, } \\
\mathrm{G}(\mathrm{MPa})\end{array}$ \\
\hline Aluminum alloy & 2800 & $72 \times 10^{3}$ & 0.33 & \\
PU & 1200 & 32 & 0.49 & 10.8 \\
ANSI 4340 & 7800 & $210 \times 10^{3}$ & 0.29 & - \\
Rubber & 1043 & 11.9 & 0.49 & 4 \\
\hline
\end{tabular}

The spokes in the Bridgestone model have a special curve, arranged in an interlacing fashion [7]. This enables them to flex effectively without twisting while providing the strength of pneumatic tires. Resilient Technologies have hexagonal honeycomb spokes, made of cellular materials employed in various applications, and particularly in the design of light-weight structures. They also have high stiffness and 
high resilience [8]. The fourth model is similar to Bridgestone curved spokes but it the same hub diameter as the honeycomb and spoke pairs models. This model has a special curve generated as a result of the hub diameter change, as shown in Figure 2(d). The purpose of the spokes is to have low stiffness, so it is required to have both stiffness and resilience under cyclic tension-compression loading [5], the spokes made of PU, and to replace air-filled pneumatic tires. Finite element (FE) analysis has been utilized extensively in the simulation of tire models due to its capability to solve complicated structural behaviors combining the non-linearity of a material and geometry [3].

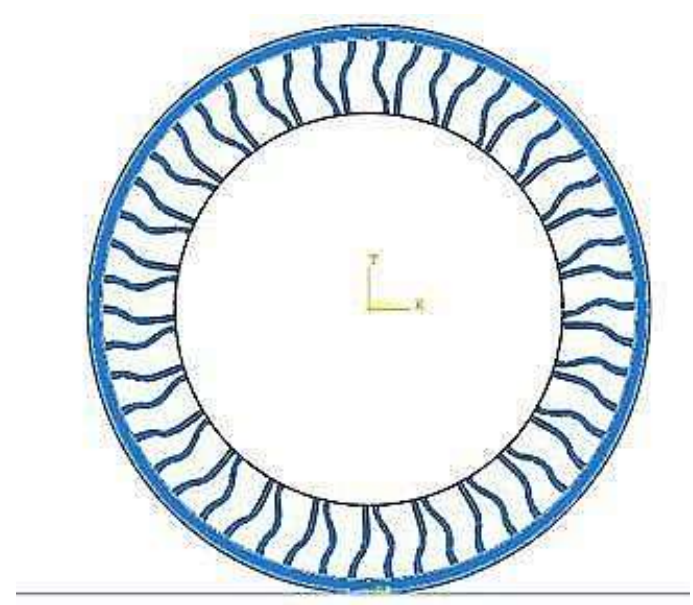

(a)

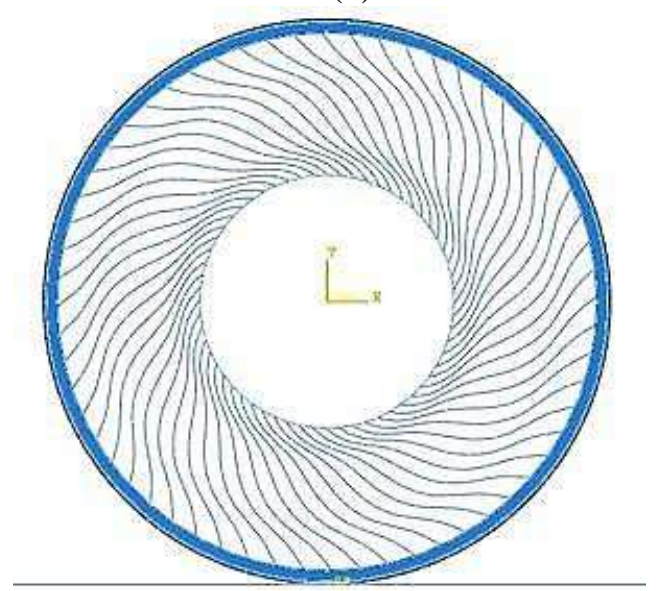

(c)

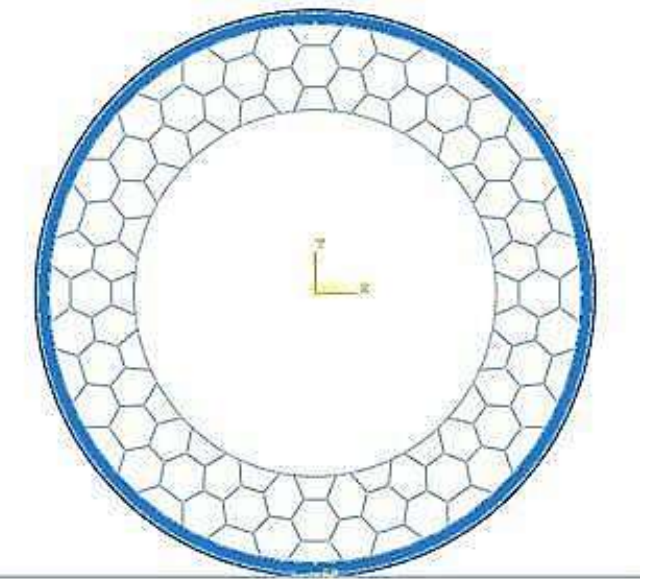

(b)

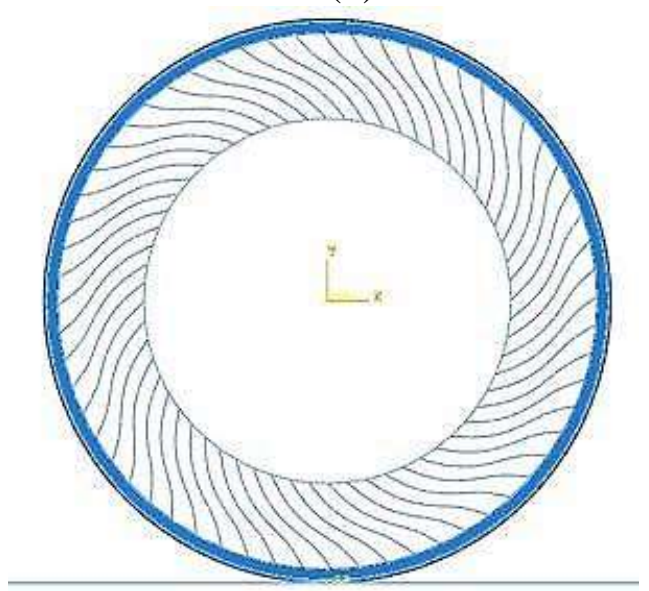

(d)

Figure 2. Four models: (a) spoke pairs, (b) honeycomb spoke, (c) curved spokec (d) New curved spoke.

\section{Geometric Parameters}

Hexagonal honeycombs are generated with the cell wall thickness, $t$, the vertical cell length, $\mathrm{h}$, the inclined cell length, 1 , and the cell angle, $\theta$, as illustrated in Figure 3. Table 2 includes the dimensions of the honeycomb cells. The effective stress-strain curves of the honeycombs vary depending on the cellular geometry. A high cell angle, $\theta$, causes cellular structures to have flexibility under uni-axial loading. The honeycomb spokes with a higher cell angle magnitude show lower local stresses, which is good for a fatigue-resistant spoke design [3]. For Tweel, the number of spoke pairs in the model is 12 with a spoke thickness $\left(\mathrm{T}_{\mathrm{s}}\right)$ of $3 \mathrm{~mm}$ each (this value for all models) and a spoke 
curvature (C) of $5 \mathrm{~mm}$ [9-11] as illustrated in Figure 4(a). The Bridgestone NPT features an inner spoke system with a special curve. The curve like a wave comes from repeated circumferentials of the hub at equal distances like the image in Figure 4(b).
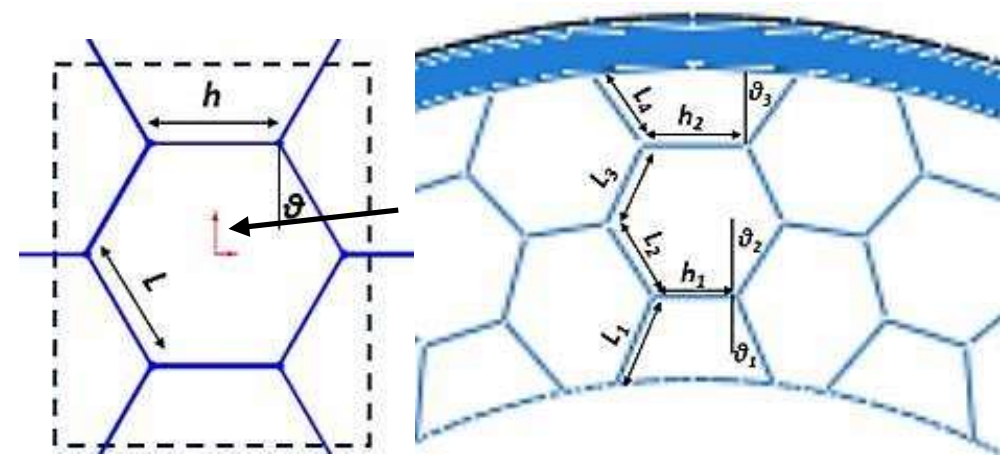

Figure 3. Geometric parameters of hexagonal honeycombs.

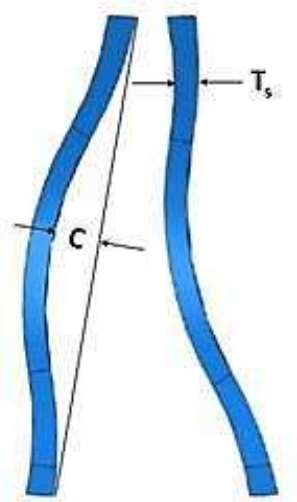

(a)

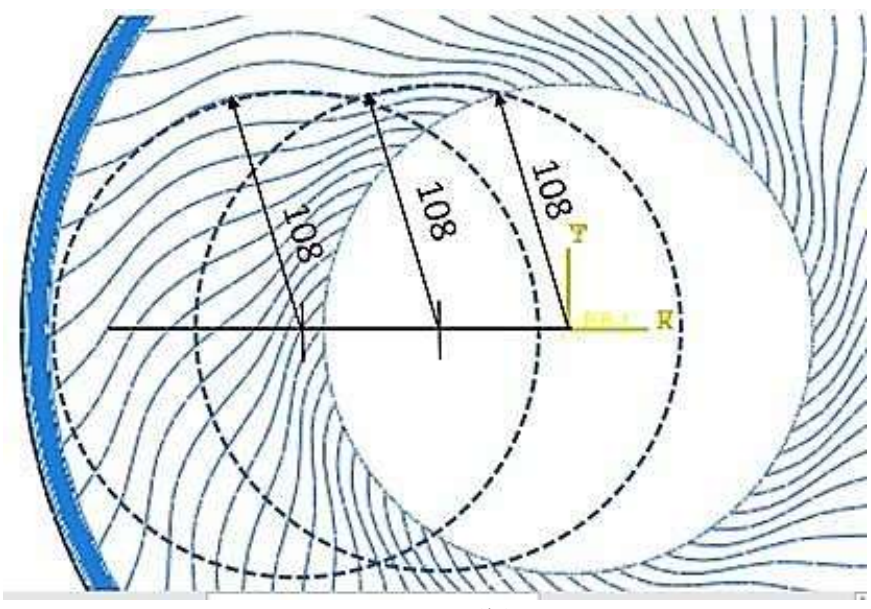

(b)

Figure 4. Geometric parameters of (a) Tweel spoke pairs, (b) spokes in Bridgestone NPT.

Table 2. Dimensions of honeycomb spoke model.

\begin{tabular}{cccccccccc}
\hline Geometric parameters & $\mathrm{L}_{1}$ & $\mathrm{~L}_{2}$ & $\mathrm{~L}_{3}$ & $\mathrm{~L}_{4}$ & $\mathrm{~h}_{1}$ & $\mathrm{~h}_{2}$ & $\theta_{1}$ & $\theta_{2}$ & $\theta_{3}$ \\
\hline $\mathrm{mm}$ (degree) & 22.5 & 20.8 & 20.5 & 21.5 & 20 & 25 & 22 & 32 & 35 \\
\hline
\end{tabular}

COMPUTATIONAL MODELING

ABAQUS/CAE 6.11, a commercial Finite Element Analysis (FEA) tool, is used to investigate the structural behavior. All interfaces, the spokes and the IIEM, IIEM and shear layer, shear layer and OIEM and the OIEM and the tread, are modeled with the tie constraint in ABAQUS. The spokes and the reference point of the hub are constrained with kinematic coupling. Quasi-static analysis that does not include mass and inertial 
effects is performed to study the time-dependent visco-elastic material response of the NPT. The analysis consists of two steps, namely the Static general and the Visco Roll. The step sequence provides a convenient way to capture changes in the loading and boundary conditions of the model. The structural analysis of the NPTs is conducted in a static general step. Material non-linearity exists in the model and geometric nonlinearity is expected during analysis. A vertical force of $3000 \mathrm{~N}$ is applied at the center of the wheel. This force corresponds to the quarter weight of the vehicle. The outer edge of the tread and the road surface are set to have contact interaction when the vertical force is applied. In the Static general step, boundary conditions are applied to stop the tire from rolling and to allow only vertical movement at the hub center, $U_{1}=0, U_{2}=$ free, $\mathrm{UR}_{3}=0$ at the reference point of the hub.

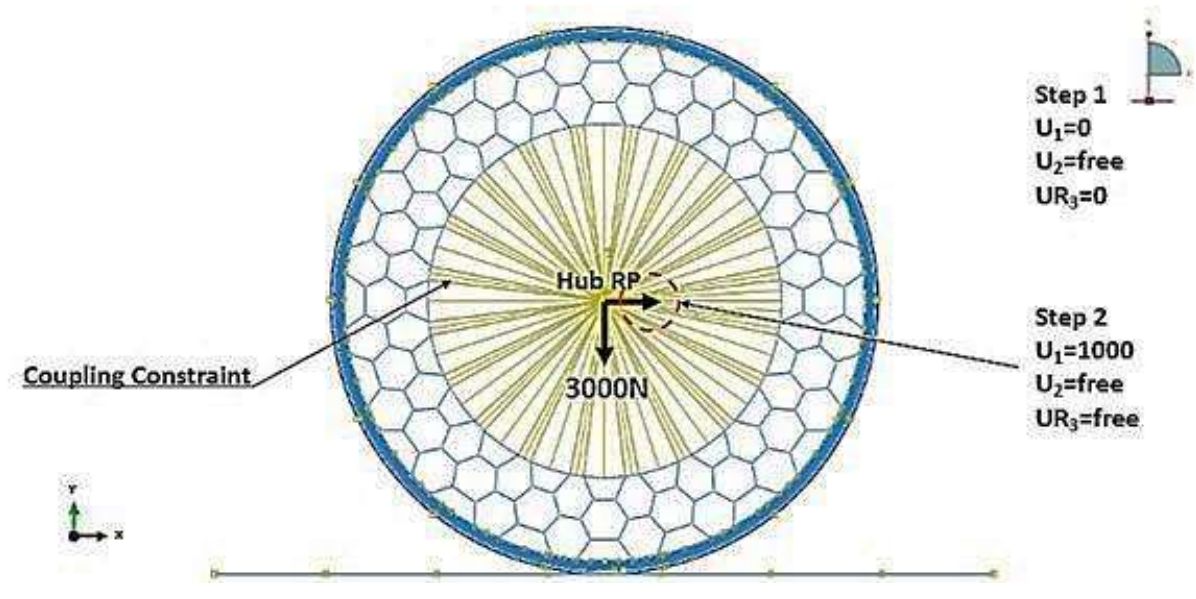

Figure 5. Interaction and boundary conditions of NPT.

In the Visco Roll step, boundary conditions are applied to allow vertical and axial movements for the tire at the hub center, $\mathrm{U}_{1}=1000, \mathrm{U}_{2}=$ free, $\mathrm{UR}_{3}=$ free, as shown in Figure 5. The ground reference point is constrained in all degrees-of-freedom (DOF) for both of the analysis steps. The (IIEM/OIEM) and spokes are modeled with shear flexible Timoshenko beam elements since beam approximation will be suitable for simulating the behavior of reinforcements and spokes. The spokes and (OIEM and IIEM) are modeled with a two-node quadratic shear flexible beam element (B22 in ABAQUS). The shear layer and the tread are modeled as CPS8R (an 8-node biquadratic plane stress quadrilateral, reduced integration) and the spokes in the Tweel model. The approximate global seed size specified in ABAQUS is 1 for all the different beam elements. The global seed size specified in ABAQUS is 0.1 for the shear layer and the tread. Uni-axial, bi-axial, and planar tension test results are shown in Figure 6(a) [7]. The three test results are used as a hyper-elastic material input of the polyurethane spokes in the FE ABAQUS code. Stress-strain behavior is also available in the literature, and is shown in Figure 6(b). It is also used for the hyper-elastic model in the FE ABAQUS code.

In fact, the Resilient Technologies NPT model does not have a shear layer and includes one reinforcement and a tread thickness larger than that of the Tweel, so two cases will be studied. In the first case all the models have the same number of parts and only the spoke shape will change according to the different types. In the second case every model will have the parts except for the shear layer and OIEM. 


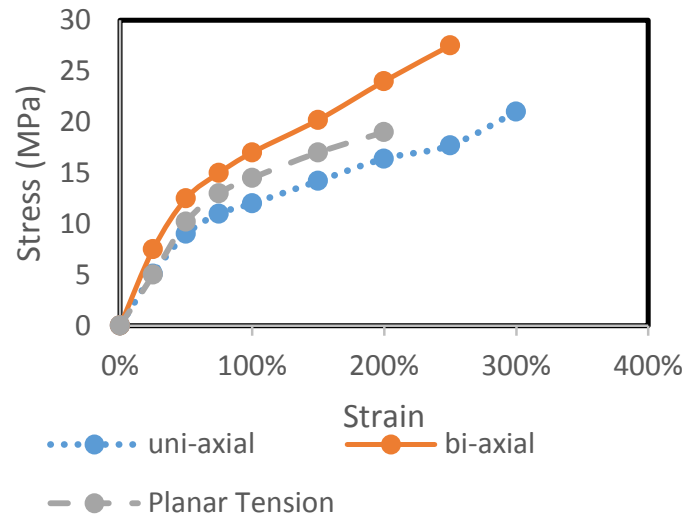

(a)

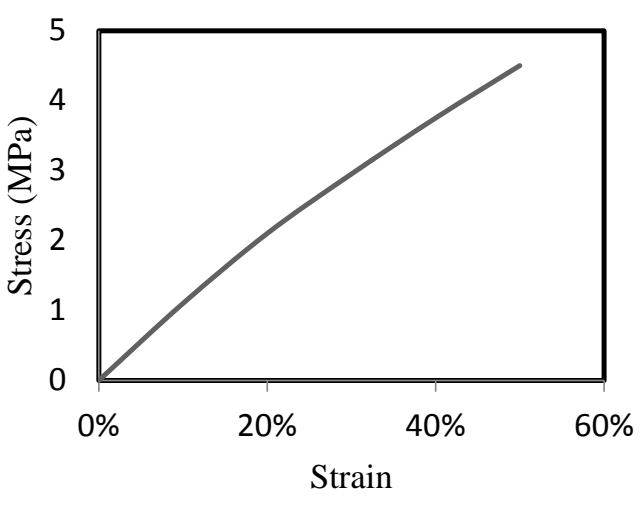

(b)

Figure 6. Stress-strain behavior of (a) polyurethane, (b) rubber.

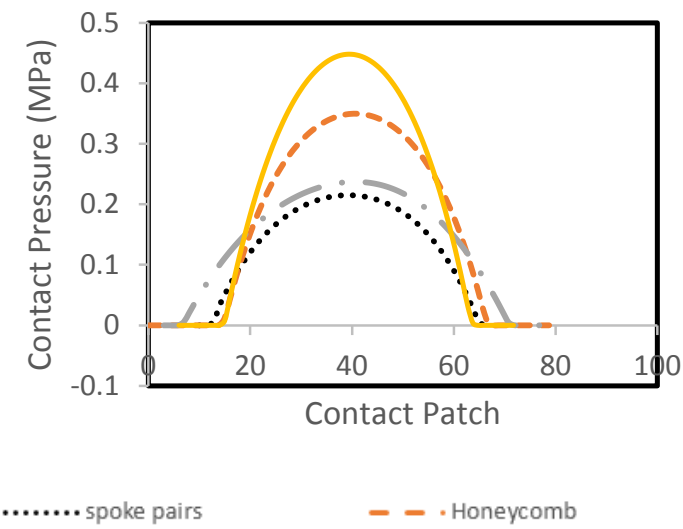

a) First case

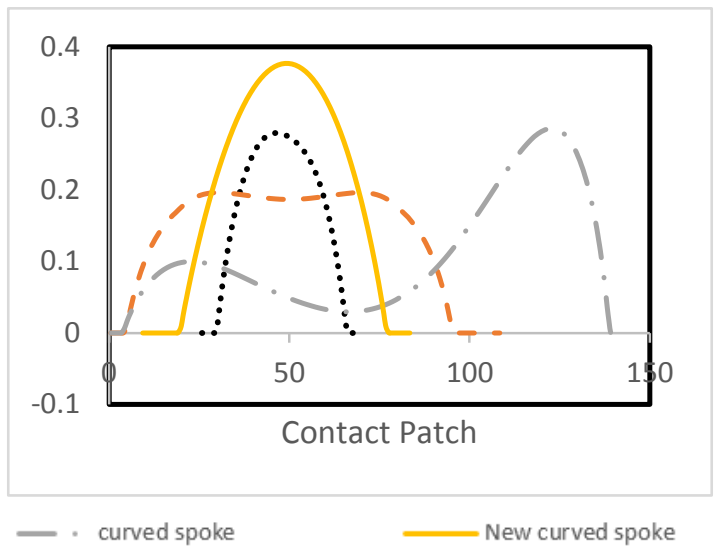

b) Second case

Figure 7. Variation in contact pressure distribution for the four models.

\section{RESULTS AND DISCUSSION}

\section{Effect of Change in Spokes Shape on Contact Pressure}

In the first case with all the models having the same number of parts, it is observed that the maximum contact pressure "CPRSS" distribution of spoke pairs has the minimum CPRSS value in the four models. An excessive distortion is observed in the curved spoke model, as the load is high for this shape. The comparison of these models is illustrated in Figure 7(a). At a vertical load of 3000N, the maximum CPRSS of the spoke pairs is $0.237 \mathrm{MPa}$ and the maximum CPRSS of the honeycomb spoke is 0.345 $\mathrm{MPa}$, and for the curved spoke is $0.215 \mathrm{MPa}$. The new curved spoke has the maximum CPRSS value of $0.447 \mathrm{MPa}$ without any distortion. In the second case, with all models not having a shear layer, including one reinforcement, and the tread thickness of 12.2 $\mathrm{mm}$, it is observed that the maximum CPRSS of the honeycomb spoke has the minimum CPRSS value in the four models. A comparison of these models is illustrated in Figure 7(b), from which it is seen that the pressure distribution has a non-uniform shape. This is due to the excessive distortion of the spokes under the applied load. 

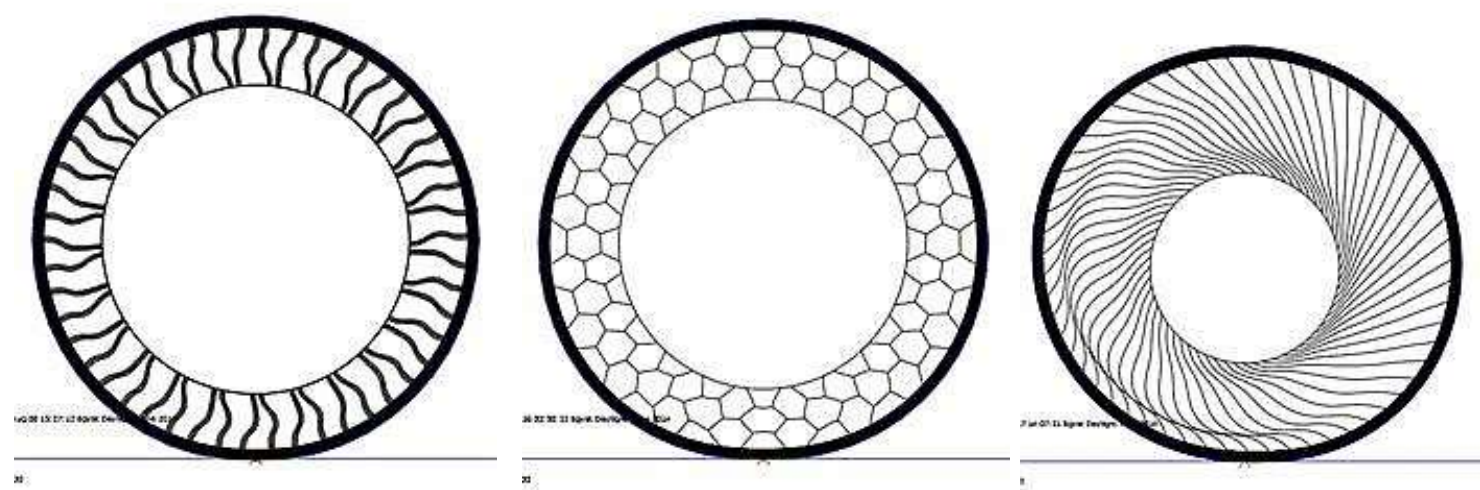

(a) Tires with shear beam
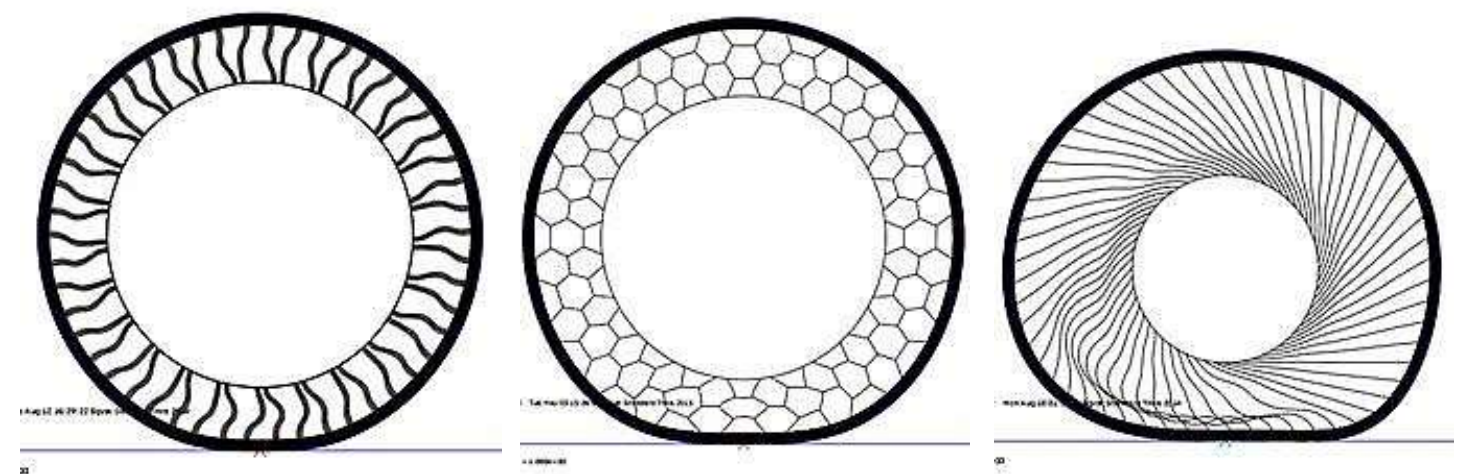

(b) Tires without shear layer and OIEM
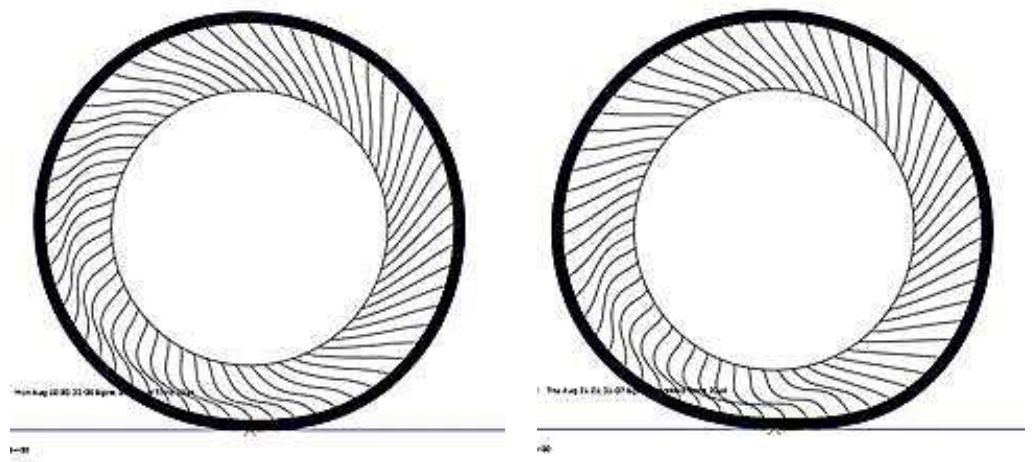

(c) New curved spoke model with shear beam (left side) and without shear layer and OIEM (right side)

Figure 8. Deformed shapes of the four models: (a, c) with shear beam and (b, c) without shear layer and OIEM.

The CPRSS results for the four models in the two cases are illustrated in Figure 8. With a vertical load of $3000 \mathrm{~N}$, it is observed that the tire with the honeycomb spoke has a maximum CPRSS of $0.196 \mathrm{MPa}$ and an excessive distortion is observed in the curved spoke model, as the load is high for this shape and the maximum CPRSS is $0.380 \mathrm{MPa}$. The maximum CPRSS of the spoke pairs is $0.285 \mathrm{MPa}$. The new curved spoke has a little distortion with a maximum CPRSS of $0.376 \mathrm{MPa}$. 


\section{Effect of Change in Spokes Shape on Von Mises Stresses}

As shown in Figure 9, the values of maximum Von Mises stresses in reinforcements in the first case are $176 \mathrm{MPa}$ for spoke pairs, $266 \mathrm{MPa}$ for the new curved spoke, $420 \mathrm{MPa}$ for the honeycomb spoke, and $479 \mathrm{MPa}$ for the curved spoke. These stresses are well below the yield strength of the aluminum alloy (7075-T6), which is $500 \mathrm{MPa}$. In the second case, the values of maximum Von Mises stresses are $396 \mathrm{MPa}$ for spoke pairs, $496 \mathrm{MPa}$ for the new curved spoke, $463 \mathrm{MPa}$ for the honeycomb spoke, and $620 \mathrm{MPa}$ for the curved spoke. The Von Mises stresses induced in the curved spoke are greater than the yield strength of the aluminum alloy (7075-T6), and this explains the excessive distortion which occurs in this model [12].

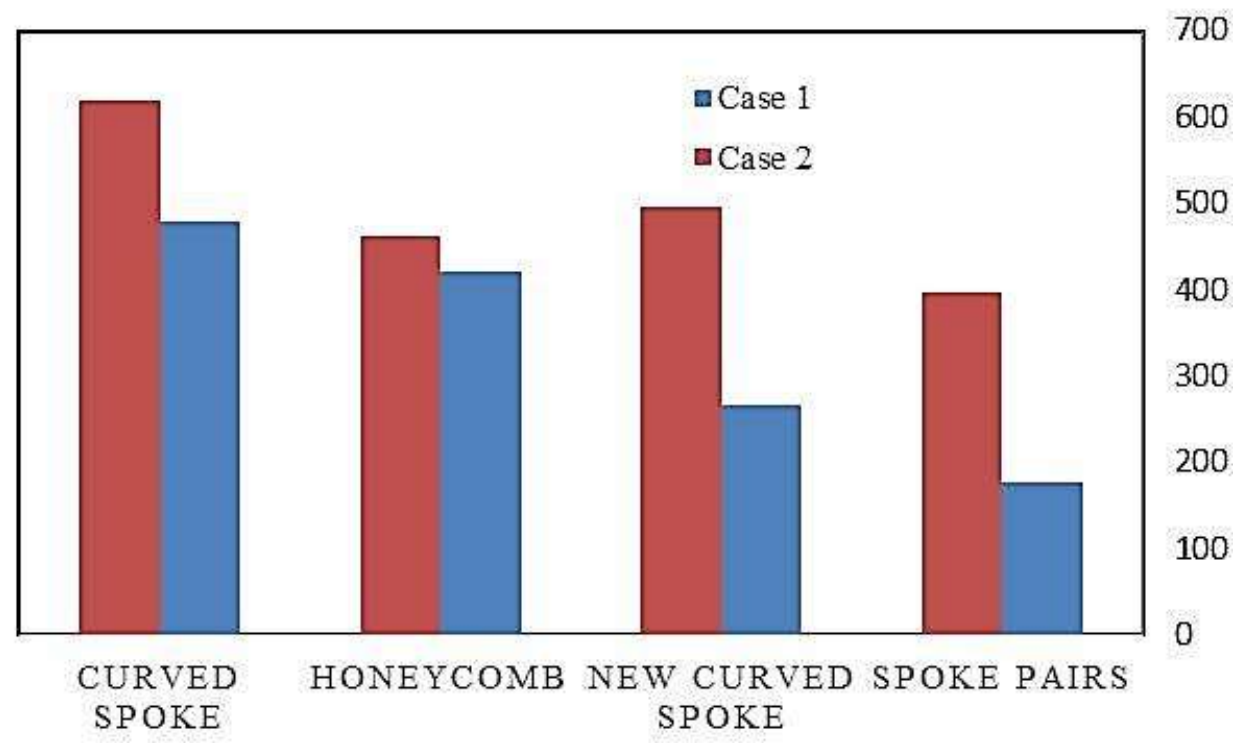

Figure 9. Comparison between the maximum Von Mises stresses of the four tire models.

\section{Effect of Change in Spokes Shape on Vertical Stiffness}

The Static Load Deflection procedure determines the displacement of the hub center upon the application of load to determine the stiffness of the tires, where vertical stiffness is defined by Eq. (1).

$$
K=\frac{F}{\partial}
$$

where $F$ is the vertical force $(3000 \mathrm{~N}) ; \delta$ is the vertical deflection of the hub center.

The tire model is compressed against the ground and hence the displacement of the hub center in the vertical direction is determined. Based on Eq. (1), the vertical stiffness of the four tire models arranged from lower to upper values is given in Table 3 , and represented furthermore as a column chart in Figure 10. As is obvious in the second case, the contact pressure and the vertical stiffness of the non-pneumatic wheel are decoupled, unlike the pneumatic structure, where they are interdependent. In this new proposed structure, combinations like high contact pressure / low stiffness and low contact pressure / high stiffness can be achieved [1, 10, 13]. 


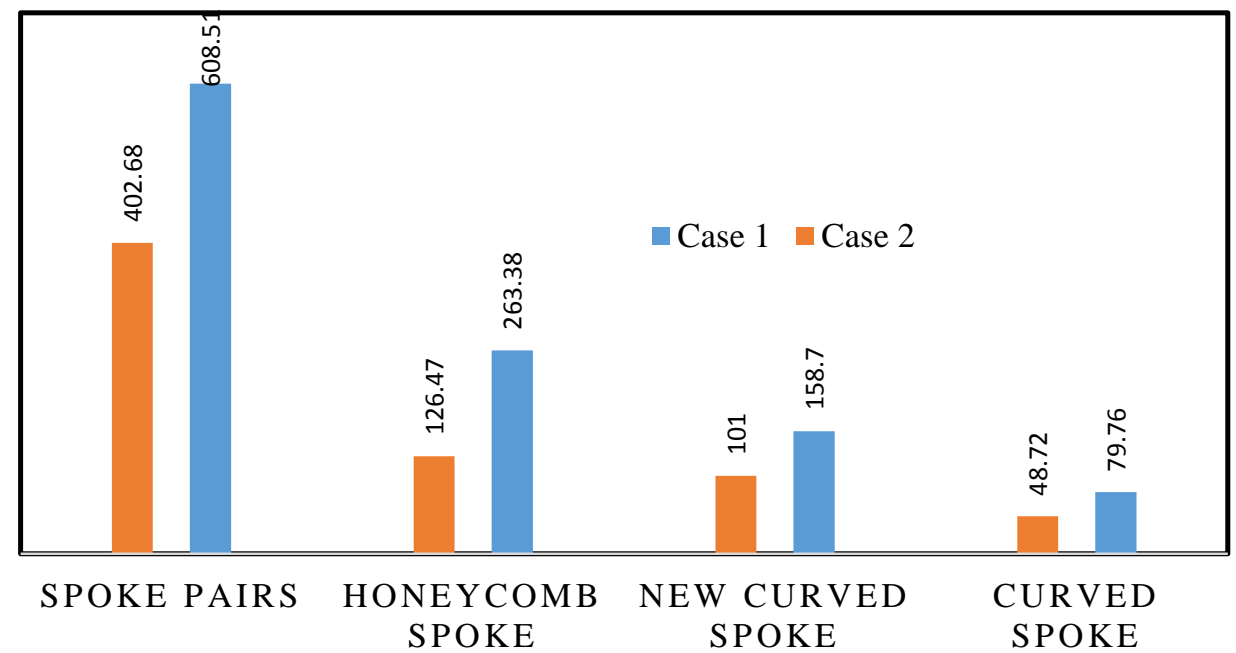

Figure 10. Comparison of vertical stiffness of the four tire models.

Table 3. Vertical stiffness for the four models.

\begin{tabular}{ccccc}
\hline \multirow{2}{*}{ Tire model } & \multicolumn{2}{c}{ First case } & \multicolumn{2}{c}{ Second case } \\
\cline { 2 - 5 } & $\begin{array}{c}\text { Deflection, } \\
(\mathrm{mm})\end{array}$ & $\begin{array}{c}\text { Vertical stiffness, } \\
(\mathrm{N} / \mathrm{mm})\end{array}$ & $\begin{array}{c}\text { Deflection, } \\
(\mathrm{mm})\end{array}$ & $\begin{array}{c}\text { Vertical stiffness, } \\
(\mathrm{N} / \mathrm{mm})\end{array}$ \\
\hline Curved spoke & 37.61 & 79.76 & 61.57 & 48.72 \\
New curved spoke & 18.9 & 158.7 & 29.7 & 101 \\
Honeycomb & 11.39 & 263.38 & 26.93 & 111.39 \\
Spoke pairs & 4.93 & 608.51 & 7.45 & 402.68 \\
\hline
\end{tabular}

\section{Total Mass of NPTs}

Figure 11 shows the total mass of NPTs in the first and second cases respectively for a lateral width of $200 \mathrm{~mm}$. It can be seen that the curved spoke has $16.67 \mathrm{~kg}$ in the first case, and $14.4 \mathrm{~kg}$ in the second case. The new curved spoke and spoke pairs have nearly the same values in the two cases, with $12.2 \mathrm{~kg}$ in the first case and $10 \mathrm{~kg}$ in the second case. The honeycomb spoke type has the lowest mass in both cases, $11.2 \mathrm{~kg}$ in the first case and $8.9 \mathrm{~kg}$ in the second case.

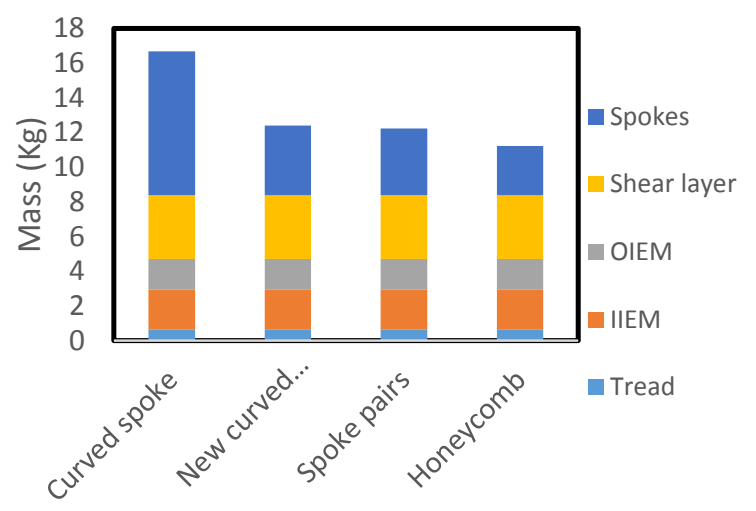

a) First case

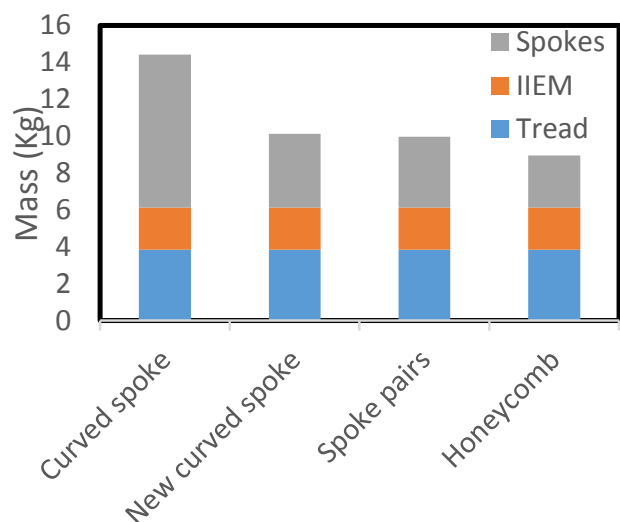

b) Second case

Figure 11. Total mass of the four models. 


\section{Rolling Resistance}

The rolling resistance (RR) can be defined by the energy dissipated or energy lost per distance rolled [4].

$$
F_{R}=\frac{W_{d}}{D}
$$

where $F_{R}$ is the rolling resistance, $W_{d}$ is the energy dissipated or energy lost, $D$ is the distance rolled by the tire.

The energy loss $W_{d}$ is numerically measured from the "ALLCD" history output in ABAQUS. ALLCD is the energy dissipated due to the creep effect of the viscoelastic materials. Shear relaxation is defined for PU and rubber. Hence the ALLCD includes energy loss in the spokes, shear layer and the tread. The time domain viscoelastic material model is active only during a transient static analysis (quasi-static analysis) and is defined for finite strain applications, where the rate-dependent elastic response is defined and the hyper-elastic material models' linear elastic isotropic material behavior is defined by a shear relaxation modulus expressed in terms of a Prony series given by Eq. (3):

$$
G_{R}(t)=G_{0}\left(1-\sum_{K=1}^{N} \overline{\mathrm{g}}_{i}^{p}\left(1-e^{-t / \tau_{i}}\right)\right.
$$

where $G_{R}(t)$ is the shear relaxation modulus, $G_{0}$ is the instantaneous shear modulus of a material, and $\mathrm{g}_{\mathrm{i}}^{\mathrm{p}}$ and $\tau_{\mathrm{i}}^{\mathrm{p}}$ are parameters used to fit the experimental data.

The data is given in ascending order for three terms $(\mathrm{N}=3)$ in the Prony series as shown in Table 4 [3]. The result values of "RR" are unexpected for the tire models; however, the goal is to compare the most accepted two models (spoke pairs and honeycomb spoke) so that the important is the percentage of those values between the models. In the first case, the "RR" of the spoke pairs is 55\% lower than the honeycomb spoke, where the "RR" of the spoke pairs is $8.47 \mathrm{~N}$ while the "RR" of the honeycomb spoke is $19 \mathrm{~N}$. In the second case, the "RR" of the spoke pairs is $43 \%$ lower than the honeycomb spoke, where the "RR" of the spoke pairs is $24.3 \mathrm{~N}$ while the "RR" of the honeycomb spoke is $42 \mathrm{~N}$.

Table 4. Viscoelastic Prony series $(\mathrm{N}=3)$ constants of a PU and synthetic rubber.

\begin{tabular}{ccccc}
\hline $\mathrm{i}$ & \multicolumn{2}{c}{ Polyurethane } & \multicolumn{2}{c}{ Rubber } \\
\cline { 2 - 5 } & $\mathrm{g}_{\mathrm{i}}$ & $\tau_{\mathrm{i}}$ & $\mathrm{g}_{\mathrm{i}}$ & $\tau_{\mathrm{i}}$ \\
\hline 1 & 0.125 & 0.002 & 0.2 & 0.002 \\
2 & 0.125 & 0.02 & 0.2 & 0.02 \\
3 & 0.125 & 0.2 & 0.2 & 0.2 \\
\hline
\end{tabular}

The results of the two cases showed the following. Mass: the curved spoke has the highest mass value in both cases, while the honeycomb spoke type has the lowest mass in both cases. The new curved spoke shows the highest maximum CPRSS value in both cases, while the lowest value has been shown for the spoke pairs in the first case and the honeycomb spoke in the second case and the pressure distribution of the honeycomb spokes is more uniform than the spoke pairs in the second case. The spoke 
pairs show the highest Vertical Stiffness values in both cases, while the lowest values have been shown for the curved spokes. It can be seen that the spoke pairs have the lowest stress values in both cases. Furthermore the Von Mises Stress induced in the material is less than its strength in all models except the curved spoke model. Rolling Resistance: the spoke pairs have the lowest 'RR' value in both cases, while the honeycomb spoke has a $35 \%$ higher value than in case 2 .

\section{CONCLUSIONS}

Two-dimensional analysis of four different spoke structures (spoke pairs, curved spoke, new curved spoke, and honeycomb spoke) of non-pneumatic tires has been conducted so as to investigate the effect of spokes structure and shear layer on tire contact pressure, vertical stiffness, stress, masses, and rolling resistance. The studies have been conducted using four full NPT models with shear beam and without shear layer, and an outer inextensible membrane. Finally, based upon the first case, it can be concluded that the spoke pairs model seems to have the best shape due to its lower rolling resistance, low contact pressure, and least Von Mises stresses on its spokes. Based on the second case, it can be concluded that the honeycomb spoke model seems to have the best shape due to having the least rolling resistance, low mass value, lower and more uniform contact pressure distribution, and accepting Von Mises stresses on its spokes.

\section{ACKNOWLEDGEMENTS}

The authors would like to be obliged to Helwan University for providing laboratory facilities.

\section{REFERENCES}

[1] Gasmi A, Joseph PF, Rhyne TB, Cron SM. Development of a two-dimensional model of a compliant non-pneumatic tire. International Journal of Solids and Structures. 2012;49:1723-40.

[2] Narasimhan A. A computational method for analysis of material properties of a non-pneumatic tire and their effects on static load-deflection, vibration, and energy loss from impact rolling over obstacles: Clemson University; 2010.

[3] Ju J, Kim D-M, Kim K. Flexible cellular solid spokes of a non-pneumatic tire. Composite Structures. 2012;94:2285-95.

[4] Veeramurthy M. Modeling, finite element analysis, and optimization of NonPneumatic Tire (NPT) for the minimization of rolling resistance: Clemson University; 2011.

[5] Manibaalan C, Keshore BS, Haran JC. Static analysis of airless tyres. International Journal of Scientific and Research Publications. 2013;3: 1-4.

[6] Proddaturi A. Robust parameter design and finite element analysis for a nonpneumatic tire with low vibration: Clemson University; 2009.

[7] Kanyanta V, Ivankovic A. Mechanical characterisation of polyurethane elastomer for biomedical applications. Journal of the Mechanical Behavior of Biomedical Materials. 2010;3:51-62.

[8] Ju J, Summers JD, Ziegert J, Fadel G. Design of honeycombs for modulus and yield strain in shear. Journal of Engineering Materials and Technology. 2012;134:011002. 
[9] Bezgam S. Design and analysis of alternating spoke pair concepts for a nonpneumatic tire with reduced vibration at high speed rolling: Clemson university; 2009.

[10] Berglind L. Design tool development for cellular structure synthesis to achieve desired properties: Clemson University; 2010.

[11] Manga KK. Computational method for solving spoke dynamics on high speed rolling Tweel (TM): Clemson University; 2008.

[12] Thyagaraja N. Requirements determination of a novel non-pneumatic wheel shear beam for low rolling resistance: Clemson University; 2010.

[13] Jagadish R. A computational investigation of contact pressure for a nonpnemautic wheel with a meta-material shear band: Clemson University; 2010. 